\title{
Construction and Application of a Medical-Grade Wireless Monitoring System for Physiological Signals at General Wards
}

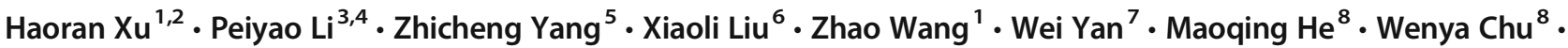 \\ Yingjia She ${ }^{9} \cdot$ Yuzhu Li $^{10} \cdot$ Desen $\mathrm{Cao}^{3} \cdot$ Muyang Yan ${ }^{7}$ Zhengbo Zhang ${ }^{3,11}$ (D)
}

Received: 7 July 2020 / Accepted: 25 August 2020 / Published online: 4 September 2020

(C) Springer Science+Business Media, LLC, part of Springer Nature 2020

\begin{abstract}
Physiological signals can contain abundant personalized information and indicate health status and disease deterioration. However, in current medical practice, clinicians working in the general wards are usually lack of plentiful means and tools to continuously monitor the physiological signals of the inpatients. To address this problem, we here presented a medical-grade wireless monitoring system based on wearable and artificial intelligence technology. The system consists of a multi-sensor wearable device, database servers and user interfaces. It can monitor physiological signals such as electrocardiography and respiration and transmit data wirelessly. We highly integrated the system with the existing hospital information system and explored a set of processes of physiological signal acquisition, storage, analysis, and combination with electronic health records. Multi-scale information extracted from physiological signals and related to the deterioration or abnormality of patients could be shown on the user interfaces, while a variety of reports could be provided daily based on time-series signal processing technology and machine learning to make more information accessible to clinicians. Apart from an initial attempt to implement the system in a realistic clinical environment, we also conducted a preliminary validation of the core processes in the workflow. The heart rate veracity validation of 22 patient volunteers showed that the system had a great consistency with ECG Holter, and bias for heart rate was 0.04 (95\% confidence interval: -7.34 to 7.42 ) beats per minute. The Bland-Altman analysis showed that $98.52 \%$ of the points were located between Mean $\pm 1.96 \mathrm{SD}$. This system has been deployed in the general wards of the Hyperbaric Oxygen Department and Respiratory Medicine Department and has collected more than 1000 cases from the clinic. The whole system will continue to be updated based on clinical feedback. It has been demonstrated that this system can provide reliable physiological monitoring for patients in general wards and has the potential to generate more personalized pathophysiological information related to disease diagnosis and treatment from the continuously monitored physiological data.
\end{abstract}

Keywords Wearable technology $\cdot$ Physiological signals · Wireless monitoring system $\cdot$ Electronic health records $\cdot$ Machine learning applications

This work was done during Zhicheng Yang's internship at Beijing Haisi Ruige Science \& Technology Co., Ltd., Beijing, China, when he was a $\mathrm{Ph}$.D. candidate at University of California, Davis, CA, USA.

This article is part of the Topical Collection on Mobile \& Wireless Health

Muyang Yan

yanmy301@sina.com

$\triangle$ Zhengbo Zhang

zhengbozhang@126.com

Medical School of Chinese PLA, Beijing, China

Medical Sergeant School, Army Medical University, Hebei, China

3 Department of Biomedical Engineering, Chinese PLA General Hospital, Beijing, China

4 Department of Computer Science and Technology, Tsinghua University, Beijing, China

5 PAII Inc, Palo Alto, CA, USA
6 School of Biological Science and Medical Engineering, Beihang University, Beijing, China

7 Department of Hyperbaric Oxygen Therapy, the First Medical Center, Chinese PLA General Hospital, Beijing, China

8 Beijing Haisi Ruige Science \& Technology Co., Ltd, Beijing, China

9 Department of Pulmonary \& Critical Care Medicine, the First Medical Center, Chinese PLA General Hospital, Beijing, China

10 Department of Pulmonary \& Critical Care Medicine, Hainan Hospital of PLA General Hospital, Sanya, Hainan, China

11 Center for Artificial Intelligence in Medicine, Chinese PLA General Hospital, Beijing, China 


\section{Introduction}

Physiological signals contain abundant personalized information. Studies have shown that vital signs can help to detect early deterioration in a general ward. In current medical practice, vital signs of patients at general wards are often measured at an interval of more than $8 \mathrm{~h}[1,2]$, only $2-4$ times per day. According to some studies, $75 \%$ of the preventable adverse events occurred in patients who were not monitored outside the ICU, and $84 \%$ of them showed signs of deterioration in the first eight hours of an adverse event such as cardiopulmonary arrest [3]. As a result of non-continuous monitoring of physiological signals and low frequency of vital sign collection, many detailed early signs of deterioration may be missed, especially at night [4]. However, the medical resources of the general ward are difficult to achieve full-time and lightweight monitoring, which is a risk for patients $[5,6]$.

With the development of wearable technology, wearable devices have the capability to monitor different physiological signals and are more suited to be deployed at general wards because of their advantages of low-cost, remote and real-time monitoring [7-10]. In addition, artificial intelligence (AI) technology has become a hot-spot in recent years. Wearable technologies based on AI have led to numerous novel functions so that many research findings of medicine based on machine learning have become available. The changes in wearable devices brought about by AI technology are summarized as follows: First, the methods of monitoring and health management have been diversified. Many wearable devices gather physiological signals via ambient wireless technologies, such as $3 \mathrm{G} / 4 \mathrm{G} / 5 \mathrm{G}$, Bluetooth, or Wi-Fi, and users can see the results and receive advice on a smartphone wherever they are [11-14]. These functions might help make users capable of basic self-management. Second, medical decision assistance can be provided with the diagnosis and prediction algorithms. The development of different algorithms and models makes some applications of diagnosis and prediction more reliable and robust using wearable devices [15-18]. Third, the effectiveness of treatment and rehabilitation can be assessed. Some patients need to have their physiological signals monitored for a long time after interventions, or have their daily state monitored in case of accidents.

Even though there are already different kinds of wearable devices and featured applications, most of the applications are still home-grade. Things may become different when they are related to clinical-grade practice, which demands better accuracy and stability. In a narrow sense, medical-grade devices can be defined as devices that be approved by the Food and Drug Administration (FDA). However, some researchers have pointed out that many wearable devices and their functions lack sufficient validation in terms of clinical accuracy and usability [19]. Furthermore, our opinion is that a medicalgrade wearable device should be validated in realistic clinical environments against traditional accepted medical grade equipment in order to prove its accuracy and feasibility. A series of previous validation studies have shown that some well-known wearable devices, such as SensiumVitals and HealthPatch, can measure physiological signals accurately when patients are in bed [4]. However, patients at general wards are not always in a relatively static state, thus throwing doubt on the accuracy of the signals measured by wearable devices. On the other hand, the high-value information hidden in physiological signals remains to be discovered. Although researchers have developed a large number of methods such as heart rate variability (HRV) analysis $[20,21] /$ multiscale entropy (MSE) [22]/Poincare plot [23, 24] to mine hidden information in the signals, the analysis and utilization of time-series physiological signals remain in its infancy. Rather than providing a bunch of data and increasing the burden of clinicians, researchers need to process what the wearable devices measure and show more further analysis results or conclusions based on evidence. Moreover, Dinh-Le et al. [9] suggested that time-series physiological signals collected by wearable devices have the potential to be combined with electronic health records (EHRs) for analysis. It is also a reminder that the analysis of physiological signals separately may be difficult and the conclusion is not reliable, so integration with EHRs may be the key to finding a way out of the dilemma facing physiological signal analysis. Demographics, lab tests, and anamnesis in EHRs can increase the dimension of information significantly and help us make better use of physiological signals measured by wearable devices.

In this paper, we presented a medical-grade wireless monitoring system that was intended to facilitate the adoption of wearable technologies in the healthcare system, which includes a multi-sensor wearable device, and a set of software and related applications by leveraging machine learning algorithms. On the basis of the system, we explored a set of continuous physiological signal acquisition, storage, analysis, and EHR combination process. The system has been deployed in the general wards in the Hyperbaric Oxygen (HBO) Department and the Respiratory Medicine Department at Chinese PLA General Hospital (PLAGH) since April 2018 and has been collecting data from patient volunteers. To our knowledge, this system is one of the earliest attempts to implement the physiological signal monitoring system in the clinic in China.

\section{Methods}

\section{System architecture}

Figure 1 is the block diagram of the system architecture. The left block exhibits the patient side of the system, which is always at general wards. The system utilizes wearable devices 


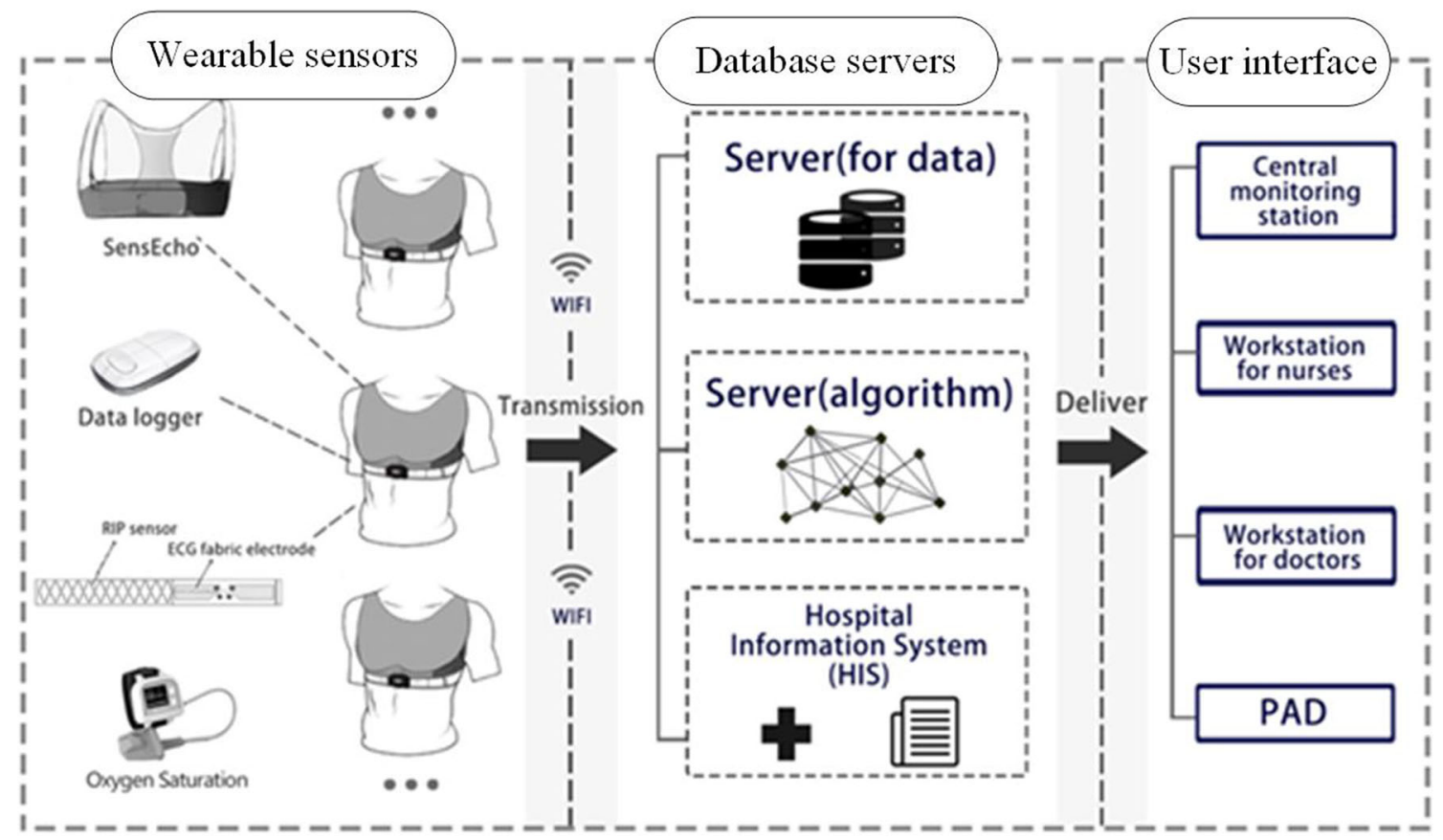

Fig. 1 Block diagram of the system architecture

to obtain patients' vital physiological signals and transmit the data to servers via Wi-Fi. The middle block shows the servers that we include in our system. The server for data is used for storage while the algorithm server can analyze the signals online based on machine learning algorithms, and we can make full use of the physiological signals in combination with the EHR information in the existing Hospital Information System (HIS) of the hospital. The right block is the user side of our system. There are many AI-based functions displayed on different terminals, including the central monitoring station, workstation for doctors/nurses, and PAD. Clinicians use their own account and password to log into the system. Information is only available to pre-registered users.

\section{Wearable sensors}

The key component of our system is the multi-sensor smart vest, SensEcho [25, 26], which has received clearance from the China FDA (Standard Number: YY 0885-2013 and GB 9706.1-2007). Sensors embedded in the vest provide ECG, respiratory and triaxial acceleration monitoring while the vest enables communication with other thirdparty wearable devices via the Bluetooth protocol. As a result, information about blood pressure, temperature, $\mathrm{SpO} 2$ can also be obtained. The vest is able to store data in the 2-GB local flash memory and/or to transmit the data to the server through Wi-Fi. Its battery supports continuous monitoring for at least $24 \mathrm{~h}$. Data generated by SensEcho can be saved online and offline. SensEcho transmits the data to servers via $\mathrm{Wi}$-Fi in real-time and can store data locally in the equipment before exporting it manually. To protect patient privacy, we use a private encrypted transport protocol. The data transmission between different devices/databases is realized on the intranet of the departments, which means data leakage is unlikely. We use an encryption method to save the local files and named as the '.CHE' file format.

ECG signal The vest provides a single-lead ECG signal at a $200 \mathrm{~Hz}$ sample rate with three fabric electrode patches embedded in the vest so that the patients only need to wear the vest that will collect the ECG signal continuously.

Respiratory signals Respiratory Inductive Plethysmography (RIP) is the core technology we used to obtain the chest \& abdominal respiratory signals, which are sampled at $25 \mathrm{~Hz}$. The vest measures chest and abdomen respiratory movement, and the output is a two-channel respiratory waveform.

Triaxial acceleration signals The signals are sampled at $25 \mathrm{~Hz}$ and the ultra-low-power sensors (ADXL345) are embedded in the vest also. Three channels of signals were measured, which stand for the movement of the patients in different directions. We can work out the posture or activity level of the patients according to these signals.

Blood pressure, temperature, $\mathrm{SpO} 2$ signals These signals are obtained by third-party wearable devices like wrist oximeter or wearable blood pressure monitors. This information is alternative. For example, patients are always asked to wear a wrist oximeter at night to measure $\mathrm{SpO} 2$ during sleep for the sake of sleep monitoring. 


\section{Data storage}

The physiological signals measured by wearable devices at a high sample rate pose a challenge to current data storage methods in a hospital, which means a new type of "Big Data". As our system is designed and implemented in a data integration approach, it is capable of dealing with heterogeneous clinical data as well as operational data. Heterogeneous data refers to data integration by various data types. In our system, the major heterogeneous data types are: 1) the timeseries physiological signals and vital signs captured by wearable devices; 2) the structured EHRs generated from daily clinical practice, such as the patient's personal information, admission records, and laboratory tests; 3 ) the unstructured clinical records, such as the medical images report and discharge summary.

We thus adopted a mixed database structure design, which has three different logical databases co-existing in the system, tackling the three corresponding data types aforementioned. The information across different databases is linked by 'patientID', 'admissionID' and 'deviceID', to guarantee the integrity of data. These three databases are elaborated on as follows.

Time-series database for physiological signals In our system, we leveraged InfluxDB (v1.7) as our time-series database. InfluxDB exploits the Time Structured Merge Tree (TSM Tree) data structure, and demonstrates superior performance in writing, querying data compression as well as scalability and usability.

Relational database for structured EHRs We adopted MariaDB as our relational database solution for the structured EHR data. The EHRs are synchronized from the HIS through RESTful application programming interface (RESTful API). The operational data, such as application's operation records, is stored in the customized schema as well.

Document-oriented database for unstructured data For the data that is not described in a relational manner, the documentoriented database, MongoDB, is used. Aside from the unstructured clinical notes, the JavaScript Object Notation formatted computation results returned from the customized applications or interfaces are stored into MongoDB as well.

\section{Functions and applications}

Functions and applications of the system are data-driven, which reflect our efforts to make the diagnosis and treatment processes more scientific. The workflow of data analysis is shown in Fig. 2. As is designed, different physiological signals, such as ECG and respiratory waves, are measured by wearable devices. In this process, preliminary analysis precedes the retrieval of information, which is relatively primitive results that can reflect much valuable information. Furthermore, features can be extracted to build some machine learning models. Limited by the analysis capabilities of current complex time-series data and sophistication of machine learning, the purpose of modeling at present is to provide more valuable information in order to support decision-making. To improve the performance of models, such EHR information as lab test results and treatment should be included in the models. Reports can be generated based on all the information available from the wearable devices. Each step in the data process can be expanded according to actually needed.

\section{Real-time monitoring}

The system can continuously monitor the physiological signals such as heart rate (HR), respiratory rate (RR), SpO2, blood pressure, posture/activities. Once a patient is admitted to the department, a nurse will scan the Quick Response (QR) code on the device and link it with the patient's information. Monitoring will begin after the nurse helps him/her put the vest on. As is shown in Fig. 3a, the real-time monitored HR, $\mathrm{RR}, \mathrm{SpO} 2$ and blood pressure are shown on the screen. Physiological parameters out of normal range will be displayed as red to raise the attention of doctors. Anomalies such as falls and arrhythmias can be detected in real-time and displayed on a warning list. As is shown in Fig. 3b, this page displays some more specific time-series information of patients. Postures, activity states, early warning score (EWS) can be calculated and displayed on the screen in real-time.

\section{Al-based application}

To facilitate machine learning technology in the system, we designed several featured AI-based applications such as sleep stage classification, sleep apnea detection, abnormal ECG signal recognition and classification and atrial fibrillation detection. These algorithms can deeply mine the hidden information in physiological signals for clinical diagnosis and treatment. We take the sleep stage classification algorithm as an example to elaborate on the data utilization when it comes to AI-based applications.

Sleep stage classification algorithm Inspired by previous studies [27, 28], we trained a deep learning model on the third party dataset then finetuned the model on our in-house dataset. We set a non-overlapping observation window for $30 \mathrm{~s}$ to extract the features, which were fed into 16-unit Bi-directional Long Short-Term Memory (BLSTM) layers and one fullyconnected layer with 4-unit outputs corresponds to the four sleep stage classes (wake, light sleep, deep sleep and rapid eye movement (REM)). The structure of the BLSTM is shown in Fig. 4. An open-access sleep database, sleep heart health study 


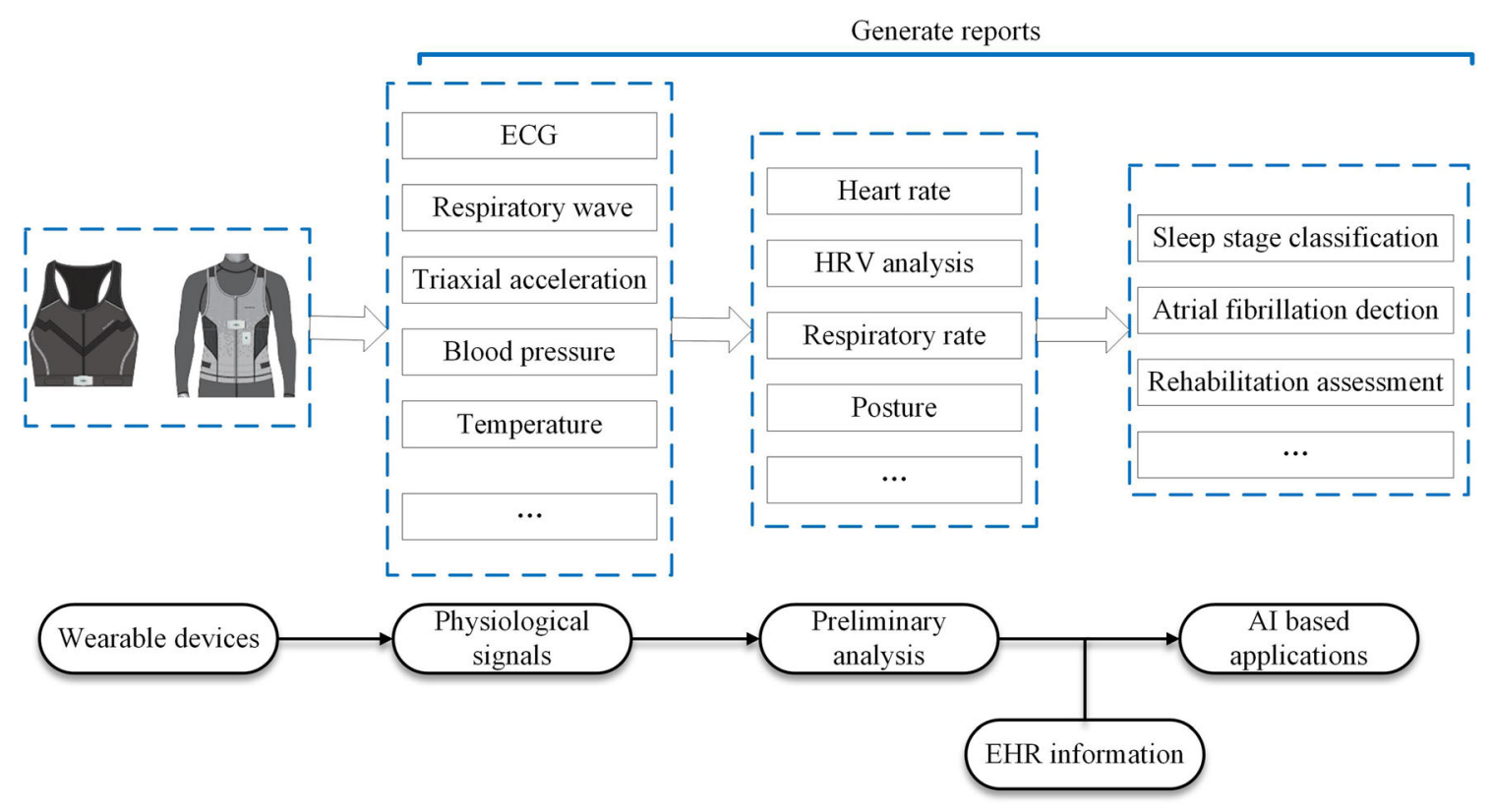

Fig. 2 The workflow of time-series physiological data analysis

(SHHS) [29], was used for model training because the volume of the in-house dataset is not big enough for the establishment of a model (about 30 labeled cases right now). The SHHS database consists of the PSG monitoring of 6600 patients in the U.S. The six-class sleep stage data for each subject manually determined by clinical experts in the database was converted into four-class (wake, light sleep (Non-rapid eye movement (NREM)-1 and NREM-2), deep sleep (NREM-3 and NREM-4), REM). Due to the limitation of the data information dimension of the training set, we only used the R-R interval and respiratory signal to extract features, the EHR information such as demographics, lab tests, drug, and anamnesis information has not been included as well. We finally extracted 152 features from R-R intervals and respiratory signals to build and train the model [30-32]. Five-fold crossvalidation was performed to test the model in the training phase, and the best model was selected to further validate the performance and we finetuned the model on the continuously collected and collated in-house dataset.

\section{Daily reports}

Based on the signal process technology and machine learning algorithms implemented in the algorithm server, we can generate a series of reports to doctors, nurses and patients daily. The types and functions of the reports are summarized as below.

Sleep quality and sleep disorder screening report Sleep quality is essential to human being's health, sleep disorder, sleep structure and sleep apnea are highly correlated with cardiovascular health $[33,34]$. Total sleep time, lasting time of the four sleep stage classes, frequency and type of sleep apneas and some other information are summarized in this report, which reflects the sleep quality of the patient.

HRV analysis report It is widely accepted that heart rate fluctuates all the time, while HRV measures the variation in beat-to-beat intervals in heart rate and is considered important for cardiac health [35]. HRV variables can be divided into three broad categories: time-domain variables, frequency domain variables and non-linear variables [36]. The HRV variables, as well as Poincare plot and detrended fluctuation analysis are summarized in this report.

Daily activity/posture analysis report Total activity time, total bedtime and energy expenditure are summarized in this report. The HR, RR and energy expenditure at each time point throughout the day can be seen in the report also. Doctors can leave recommendations below based on the report.

Other reports There are some other daily analysis reports derived from the continuous monitored physiological data (usually $24 \mathrm{~h}$ ), such as CPC analysis and breathing pattern analysis, mental stress analysis, multiple-scale analysis on heart rate and breathing rate, which are still under clinical validation and will put into clinical use when they are qualified for diagnosis and treatment purpose.

As is shown in Fig. 5, users (clinicians and nurses) can generate a report by using data in local files (also can generate reports online). Some of the reports are still under validation. The types of reports and capabilities of this function are expanding with the feedback from the clinicians and implementation of new algorithms. 
Buttons

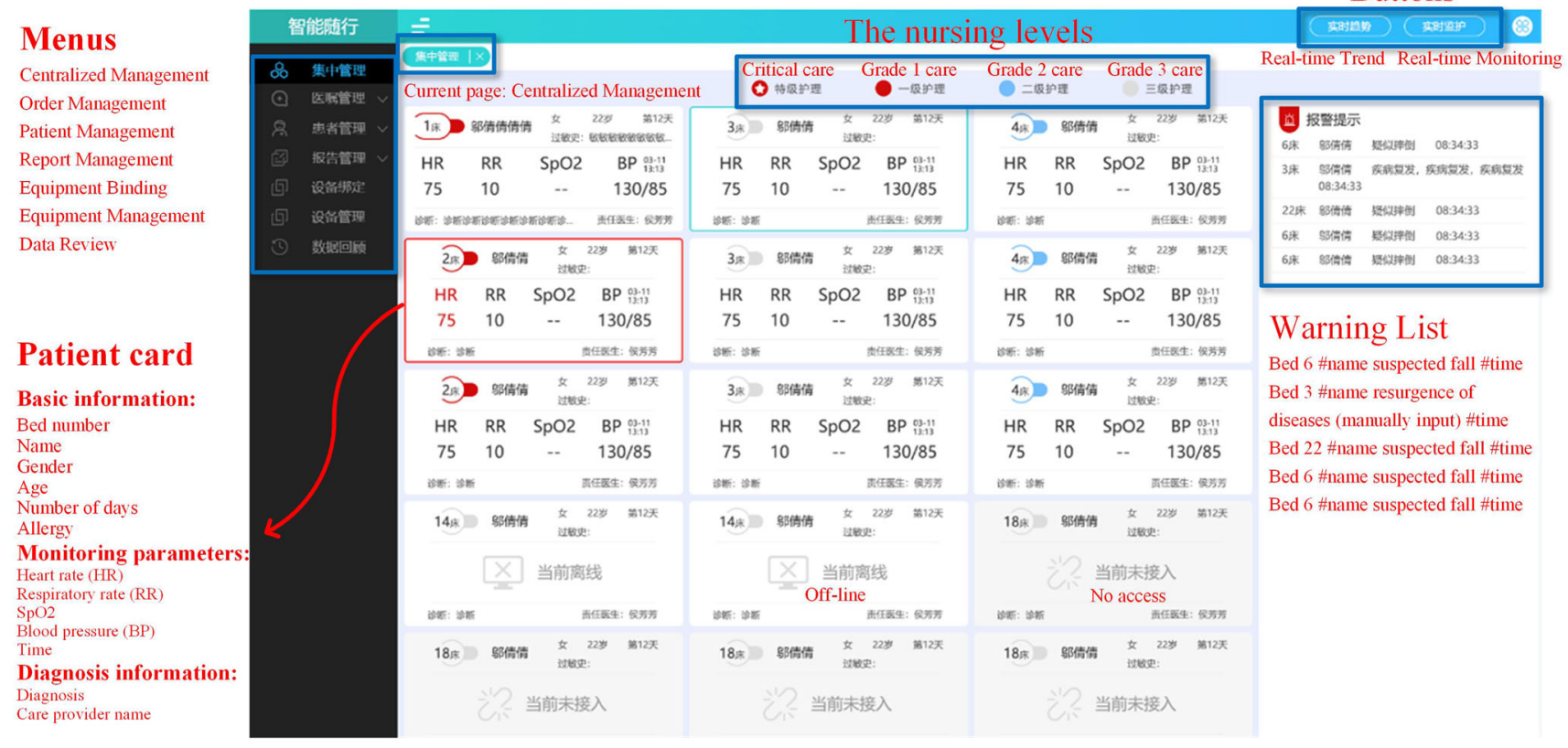

\section{a Administration \& monitoring page}

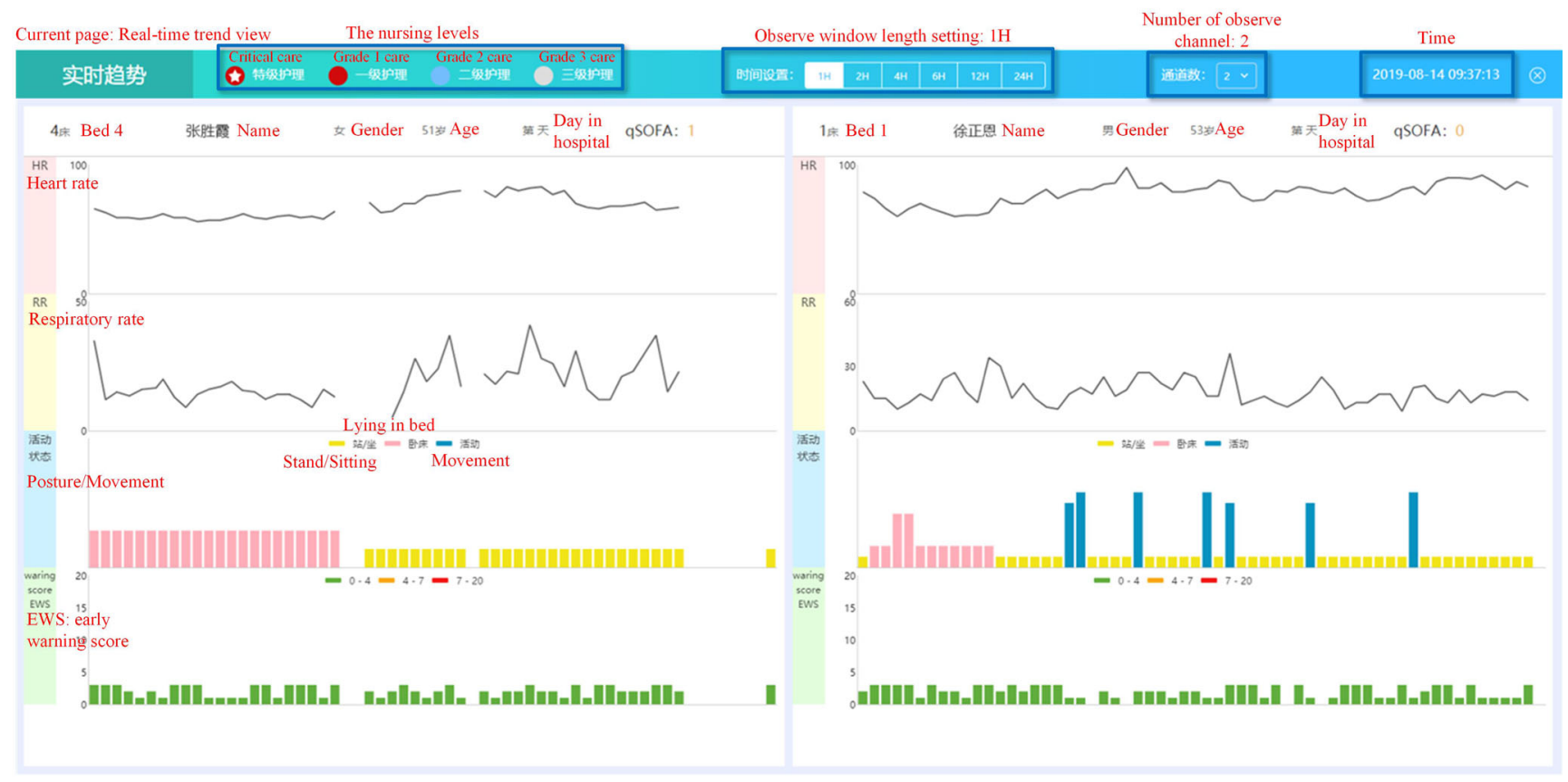

\section{b Real-time monitoring page}

Fig. 3 The real-time monitoring function of the system. a Administration \& monitoring page b Real-time monitoring page

\section{Validation experiment}

Several system performance validation experiments are underway, including the physiological signal measurement veracity and algorithm performance. In this paper, we conducted a heart rate veracity validation study, in which we compared the system with ECG Holter.

\section{Heart rate veracity validation}

Patients admitted to the HBO Department in PLAGH and subjected to 24-h continuous ECG check were informed about the objectives and risks of the experiment and inquired about their willingness to participate in the study. Volunteers were monitored on the first day of admission both by SensEcho and 


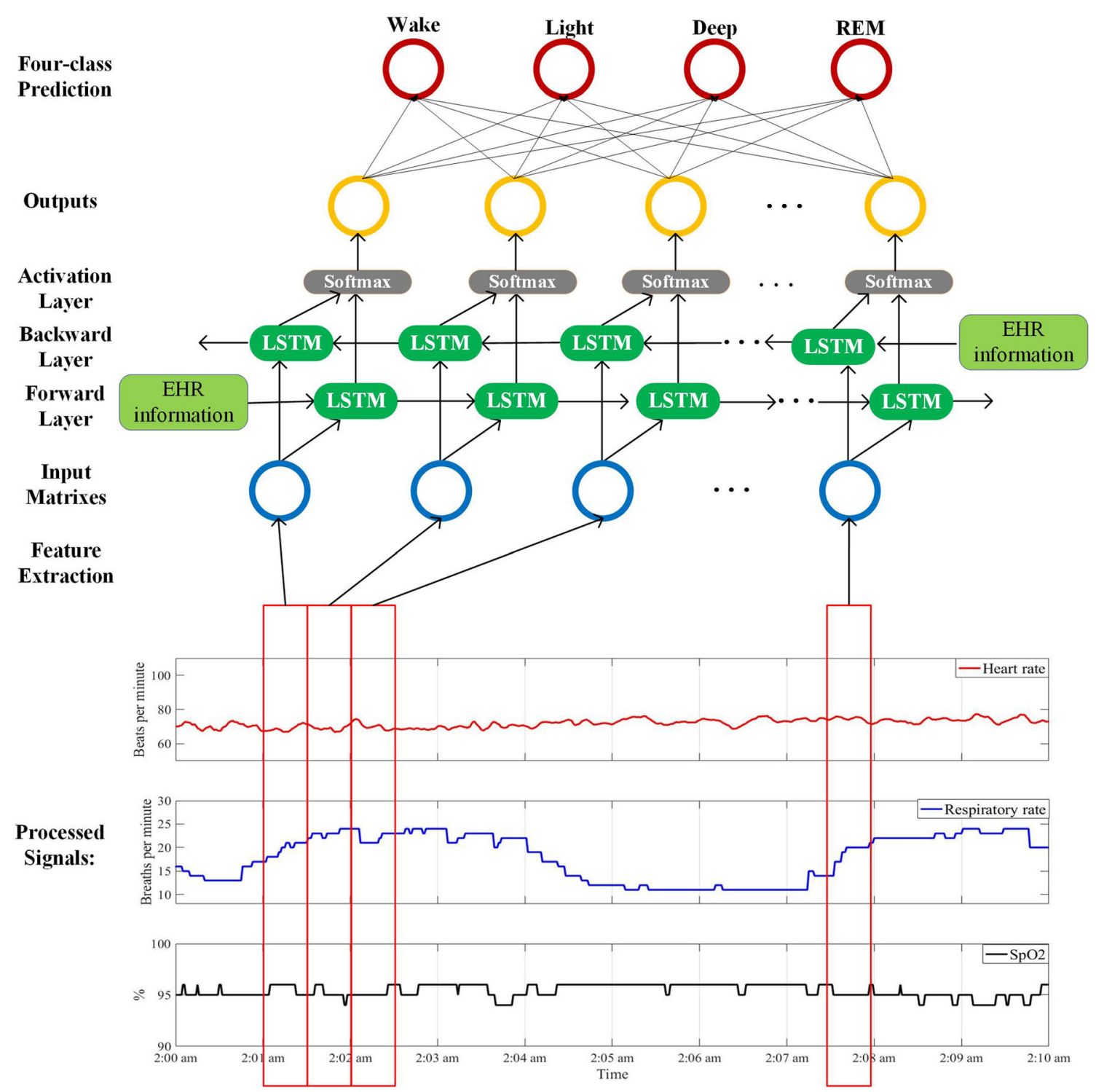

Fig. 4 BLSTM based sleep stage classification algorithm framework

Fig. 5 Report generation page (using local CHE files)
CHE文件上传 Upload CHE file page

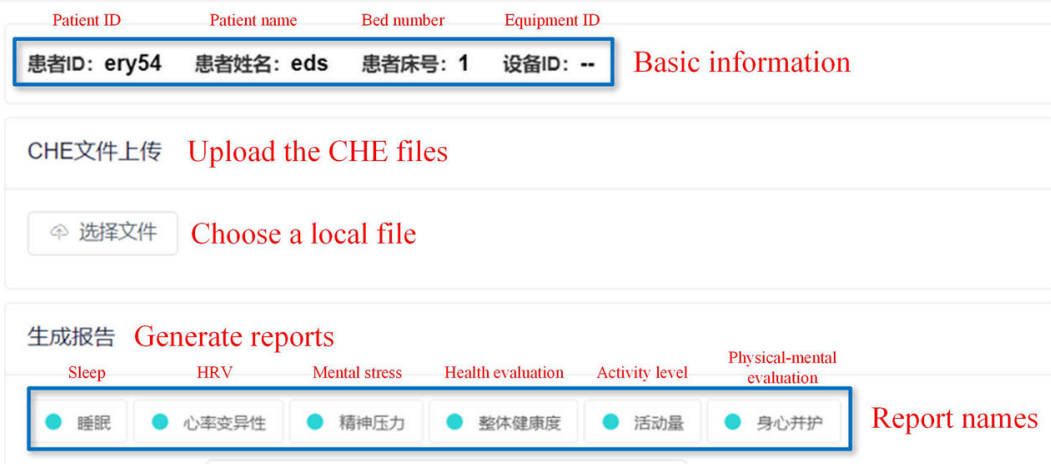

佩戴开始结束时间: 请选择闰戴开始结束时间 $\quad$ Time of the record (filled in automatically )

产生报告 Generate report button 
ECG Holter (BI6812, Biomedical Instruments Company, China. It's a type of Holter commonly used in clinical practice in PLAGH.) and they were allowed to move freely. The study was approved by the ethics committee of PLAGH.

The raw data transmitted by SensEcho contained the measurements and their timestamps while the raw ECG data gathered by ECG Holter was retrieved in TXT format. We scanned the ECG data of the two devices with the same algorithm in order to mark the $\mathrm{R}$ wave positions. Afterward, the HR was calculated according to R-R intervals. In order to reduce the impact of outliers in the signals, we set one rule that was applicable to both devices: We scanned the signal according to the $3 \delta$ principle, the time window we set was $60 \mathrm{~s}$, we calculated the mean $(\overline{\mathrm{x}})$ and standard deviation $(\delta)$ in the time window and checking if the HR value of the middle point was within $\overline{\mathrm{x}} \pm 3 \delta$, then we considered the points beyond the threshold as outliers as well. Finally, we set all of the outlier points in signals to null $(\mathrm{NaN})$ which were not included in the analysis. HR data was then resampled to once per minute before subsequent analysis. We defined $\mathrm{HR}<50$ beats per minute (bpm) as bradycardia and HR $>100 \mathrm{bpm}$ as tachycardia according to the reference standard [37-39].

To align the timeline, the offset was calculated by using the best fit of a cross-correlation of the heart rate between the two signals before the spare data was cut to make sure the experiment started and ended at the same time point.

The series of observation pairs of HR measurements derived from the SensEcho and ECG Holter were compared using the Bland-Altman Method [40]. This method compared the measurements from two devices by evaluating the relationship between the HR differences and the average at every second of the records [41].

\section{Results}

\section{HR veracity validation result}

Twenty-seven patients were enrolled in this study between March 2018 and August 2019. Data was lost for five patients due to technical issues. In addition, we expected the monitoring to last 24-h or more for each patient. However, the fact was that due to the demand for clinical practice, some of the monitoring had to be suspended ahead of time. As a result, the monitoring data obtained from the 22 patients lasted from $591 \mathrm{~min}$ to $1434 \mathrm{~min}$. The median (Q1 - Q3) monitoring time was 1411 (1338-1418) minutes. The basic information of the patients is shown in Table 1. In this table, similar characteristics of the patients in the HBO Department could be identified, who were
Table 1 Basic information of patient volunteers

\begin{tabular}{lll}
\hline Gender & MALE $(\boldsymbol{n}=\mathbf{1 4})$ & FEMALE $(\boldsymbol{n}=\mathbf{8})$ \\
\hline Demographic (mean \pm std) & & \\
Age & $51.00 \pm 9.90$ & $56.00 \pm 9.90$ \\
Height $(\mathrm{cm})$ & $170.33 \pm 9.29$ & $162.00 \pm 3.46$ \\
Weight $(\mathrm{kg})$ & $76.00 \pm 11.27$ & $65.67 \pm 8.96$ \\
BMI & $25.87 \pm 2.72$ & $23.87 \pm 2.04$ \\
Basic Diagnosis (n (\%)) & & \\
Coronary Heart Disease & $12(85.71 \%)$ & $6(75.00 \%)$ \\
Diabetes & $6(42.86 \%)$ & $2(25.00 \%)$ \\
Hypertension & $7(50.00 \%)$ & $5(62.50 \%)$ \\
Hyperlipemia & $7(50.00 \%)$ & $5(62.50 \%)$ \\
\hline
\end{tabular}

mostly overweight, middle-aged and elderly, and afflicted with senile diseases.

\section{Examples of patient measurements}

Figure 6a shows 10-min ECG and HR signals SensEcho and the Holter measured from patient NO.01, who was a 62 -year-old female patient with extensive cardiac and vascular comorbidities like hypertension, hyperlipemia, arrhythmia. The red dots in the subplot $1 \& 2$ of Fig. 6a mark the $\mathrm{R}$ peaks in the ECG signals. Figure $6 \mathrm{~b}$ shows the same periods of signals as in Fig. 6a, a respiratory wave is measured from the chest by SensEcho. From the respiratory wave and triaxial acceleration signals, we can find that the patient was quiet and the increase of HR was not caused by movement. Afterward, the increase of HR was recognized as tachycardia by a clinical expert that lasted about 4-min and the following two spines in Fig. $6 \mathrm{~b}$ were two premature beats. The time tachycardia occurred was about 5:30 am and such clinically abnormal events are often ignored by clinicians or patients. The Fig. 6c shows the entire HR signals of this patient monitored by SensEcho and Holter respectively, which consist of $80,513 \mathrm{~s}$ of recording. It can be seen from Fig. 6 that the two systems have good consistency in terms of ECG recording and heart rate calculation.

\section{HR comparison}

For analysis, 468.98-h of HR data from 22 patients was available and the measurement pairs were 28,374 . The mean bias (Holter minus SensEcho) in HR was $0.04 \mathrm{bpm}$ with a $95 \%$ confidence interval (CI) of -7.34 to $7.42 \mathrm{bpm}$. Figure 7 is a box plot to show the HR measurement distribution of every patient. From Fig. 7, we can find that most of the patient volunteers showed similar performance between the two methods while the 
Fig. 6 An example of signals monitored by SensEcho and Holter in the validation study. a 10-min scope of signals Holter and SensEcho measured of patient NO.01. b HR, respiration and triaxial acceleration

SensEcho measured. c The entire HR signals of this patient SensEcho and Holter monitored
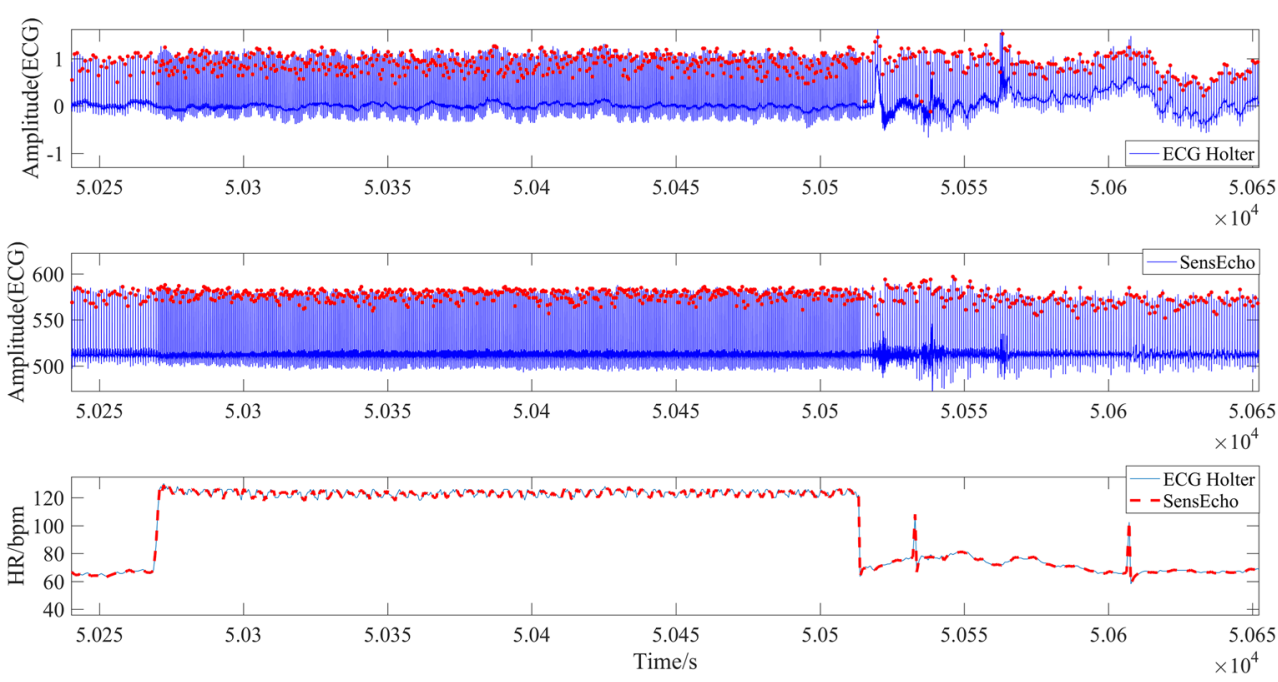

a 10-min scope of signals Holter and SensEcho measured of patient NO.01
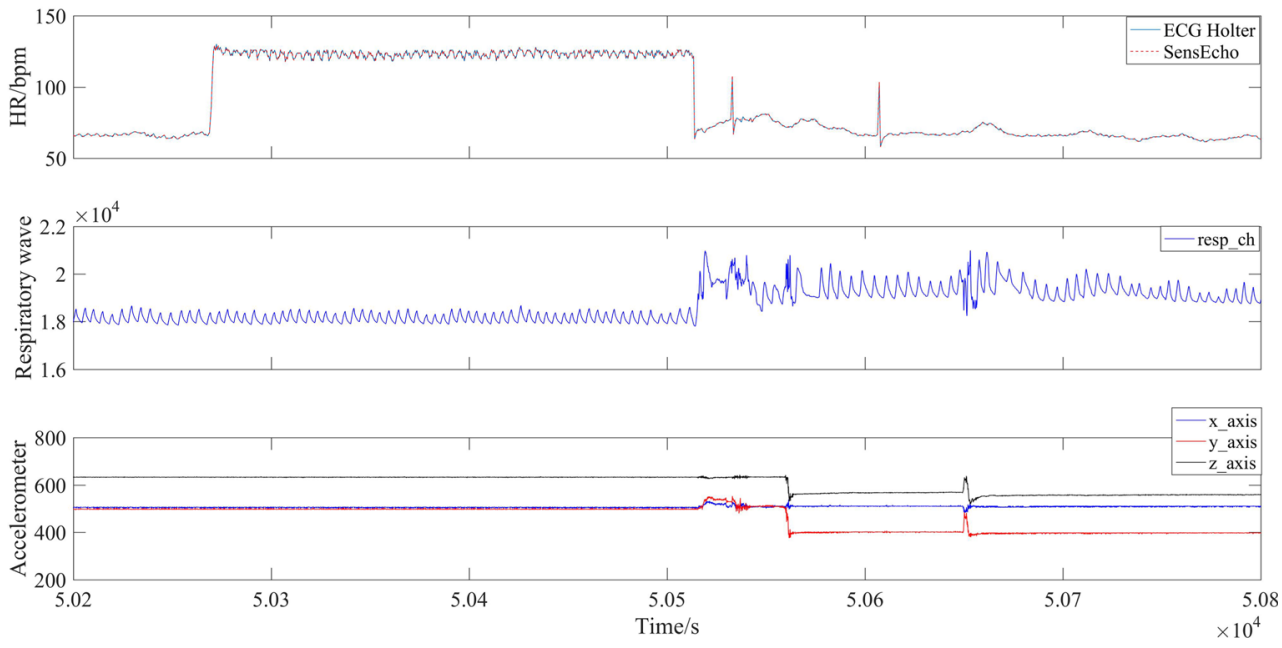

b HR, respiration and triaxial acceleration SensEcho measured
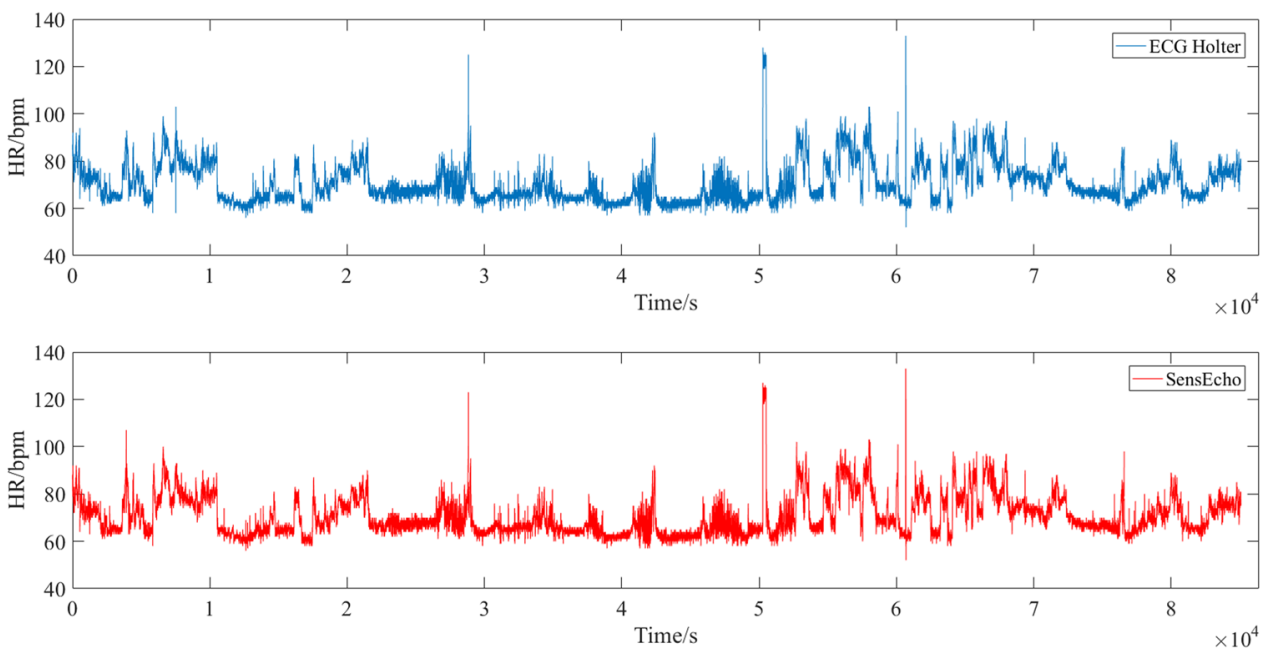

c The entire HR signals of this patient SensEcho and Holter monitored 


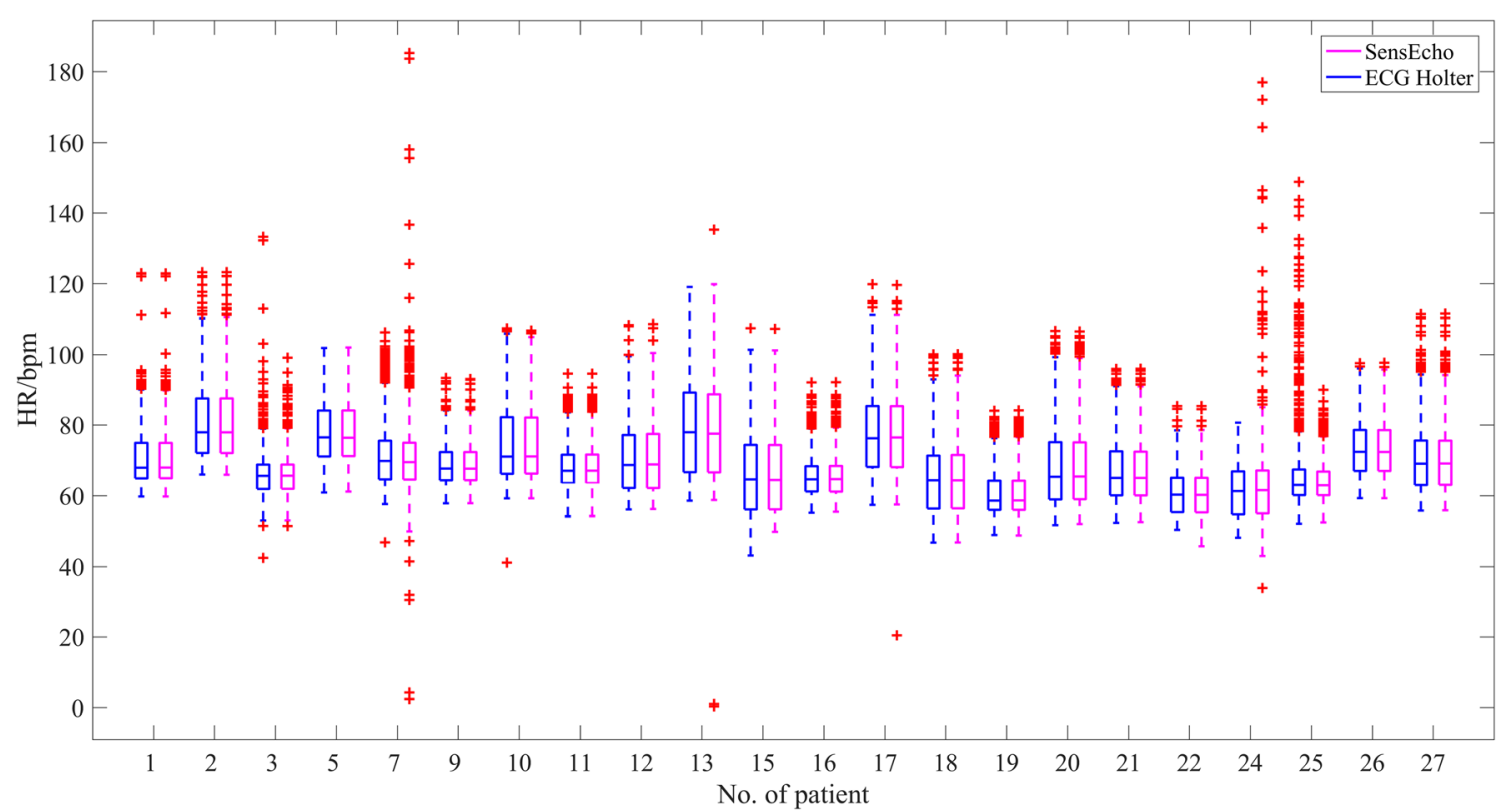

Fig. 7 Boxplot of the HR measurement distribution of the two methods for every patient

difference that may have been caused by the wearable vests that were not the right size rigorously. Figure $8 \mathrm{a}$ is the Bland-Altman plot of the whole dataset. The analysis showed that $98.52 \%$ of the points were located between Mean \pm 1.96 SD. From Fig. $8 \mathrm{~b}$ we can find that the BlandAltman plot shows great agreement between the two measurement methods, but the SensEcho is more likely to produce measurements that are too high or too low compared to the Holter. However, these measurements are quite unlikely, for there are only very few points located outside Mean $\pm 1.96 \mathrm{SD}$. We can still consider the HR measurement capacity of SensEcho reliable. In addition, unbalanced measurements may be caused by the difference in hardware passbands between the two devices.

During the long-term and high-frequency sample rate of the monitoring of the high-risk patients, as we defined before, tachycardia (HR $>100 \mathrm{bpm})$ was $1.27 \%$ and $1.23 \%$ while bradycardia $(\mathrm{HR}<50 \mathrm{bpm})$ was $0.49 \%$ and $0.54 \%$ of all HR measurements for Holter and SensEcho respectively. The sensitivity, specificity, positive predictive value, and negative predictive value were calculated and are shown in Table 2.

The Bland-Altman analysis quantitatively showed that there was great consistency between SensEcho and the ECG Holter at HR measurement. The difference was relatively small and understandable. One major reason for the difference between the two devices was outliers. Although we set filtering methods to detect the outliers and reduce the impact of them, outliers were unavoidable in time series physiological signals. Furthermore, the difference between the two devices may be due to the hardware clock limitations, where $1 \mathrm{~s}$ on one device might correspond to $0.98 \mathrm{~s}$ from the other device for example [41].

\section{An example of report}

By utilization of the raw data measured from patients, processed information from preliminary analysis, AI-based applications and EHR information from HIS, we can generate various reports. Figure 9 shows an example sleep quality and sleep disorder screening report of a patient. Figure 9a shows the trend-charts where we can visually see the sleep stage classification, posture, the onset and duration of apnea/hypopnea and physiological signals of the example patient. Figure $9 \mathrm{~b}$ shows a summary of the sleep monitoring results, which includes numerical statistics of different indicators. From the example patient, we can find that the patient went to sleep at about 11:30 pm. Time of total sleep and deep sleep was lower than the reference values, the sleep structure of this patient was not very good as well. $\mathrm{SpO} 2$ declined several times and lasted for a while and sleep respiratory events occurred during sleep monitoring. The longest sleep apnea lasted for $29.0 \mathrm{~s}$ while the total sleep apnea lasted for $7 \mathrm{~min}$ and $21 \mathrm{~s} .64 .7 \%$ of sleep respiratory events were classified as obstructive sleep apnea, while $35.3 \%$ were classified as hypopnea. The apneahypopnea index (AHI) of the patient this night was 2.7 , which is lower than the clinical diagnostic criteria of clinical sleep apnea-hypopnea syndrome. Certainly, the information provided by the system is an expansion of the information available to clinicians, which should be confirmed and can be modified by clinicians in case the algorithms are misdiagnosed. 


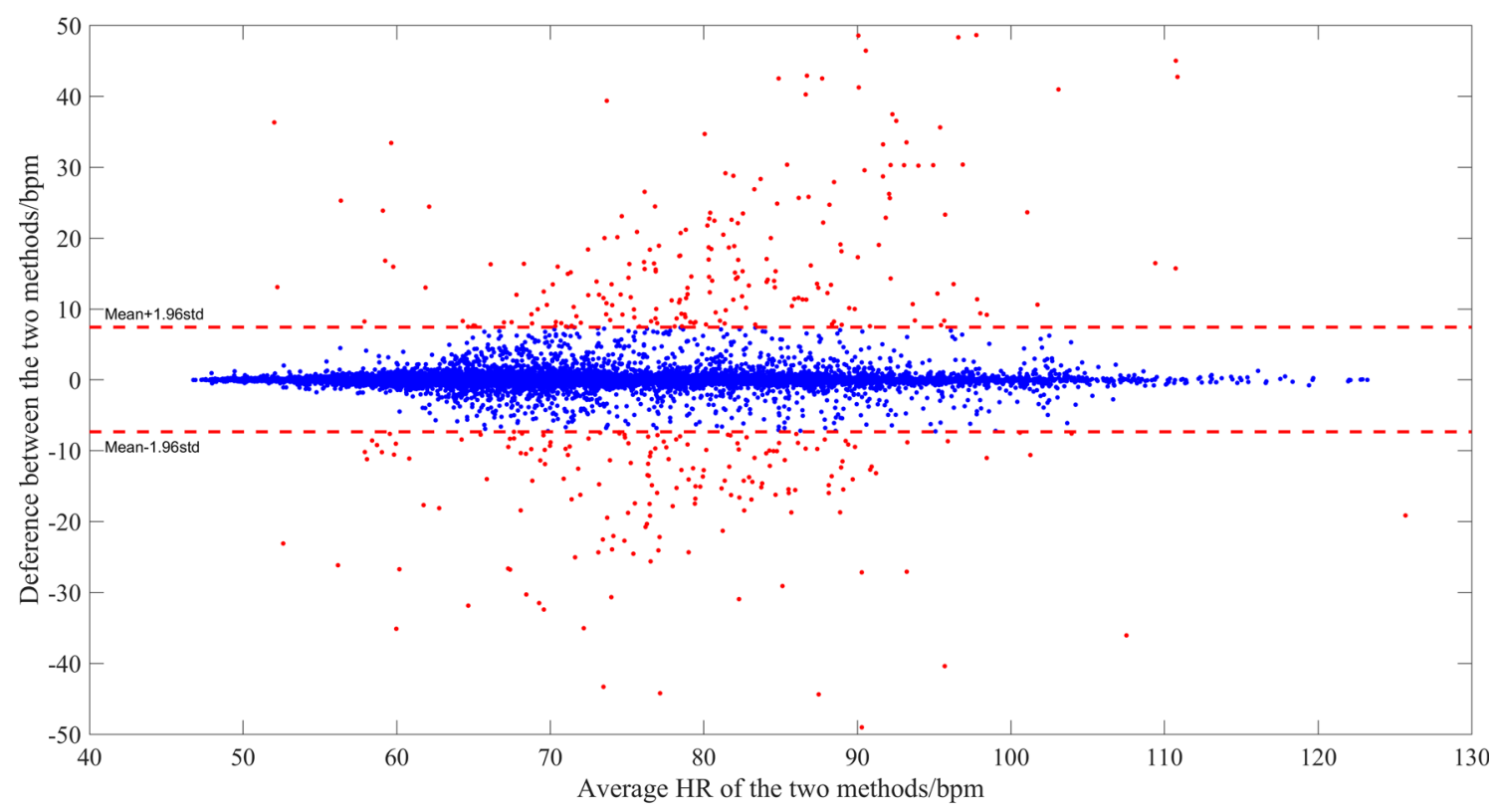

a Bland-Altman plot of the HR measured by the two methods

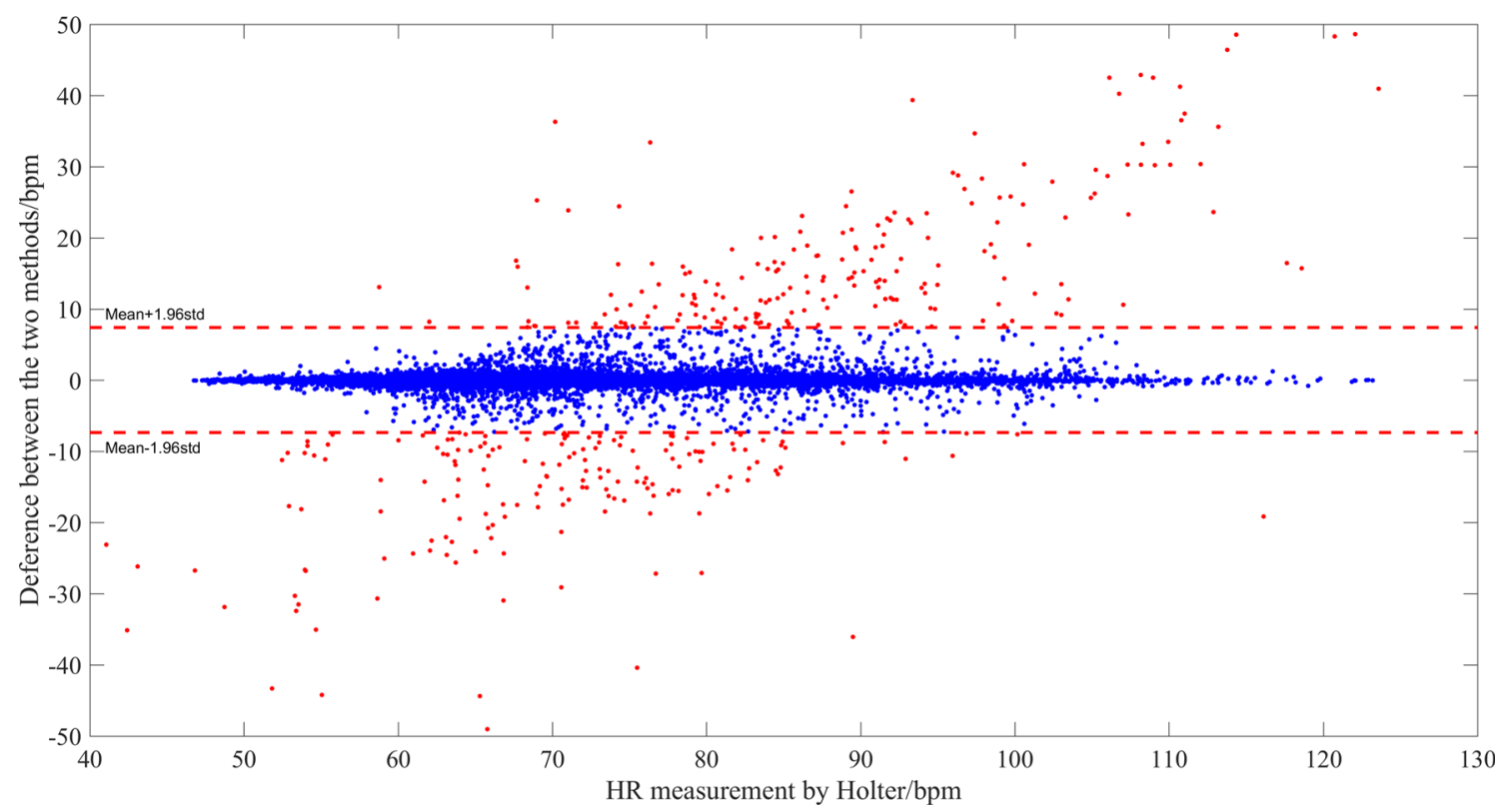

b Deviation of measurements of SensEcho against the 'golden standard', Holter

Fig. 8 Agreement analysis plots of the two methods. a Bland-Altman plot of the HR measured by the two methods. b Deviation of measurements of SensEcho against the 'golden standard', Holter

Table 2 Diagnostic accuracy for bradycardia, tachycardia (a Positive Predictive Value; b Negative Predictive Value; c F1=2 * (Precision * Recall) / (Precision + Recall)

\begin{tabular}{|c|c|c|c|c|c|}
\hline & Sensitivity (Recall) & Specificity & PPV $^{\mathrm{a}}$ (Precision) & $\mathrm{NPV}^{\mathrm{b}}$ & $\mathrm{F} 1^{\mathrm{c}}$ \\
\hline Bradycardia (\%) & 92.86 & 99.92 & 85.53 & 99.96 & 0.89 \\
\hline Tachycardia (\%) & 81.44 & 99.80 & 84.24 & 99.76 & 0.83 \\
\hline
\end{tabular}




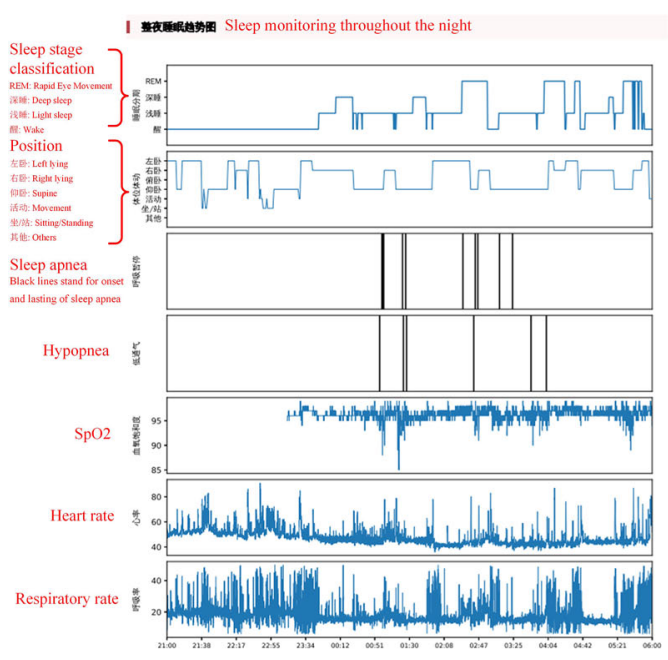

a Example patient's physiological signal trend-charts throughout the night

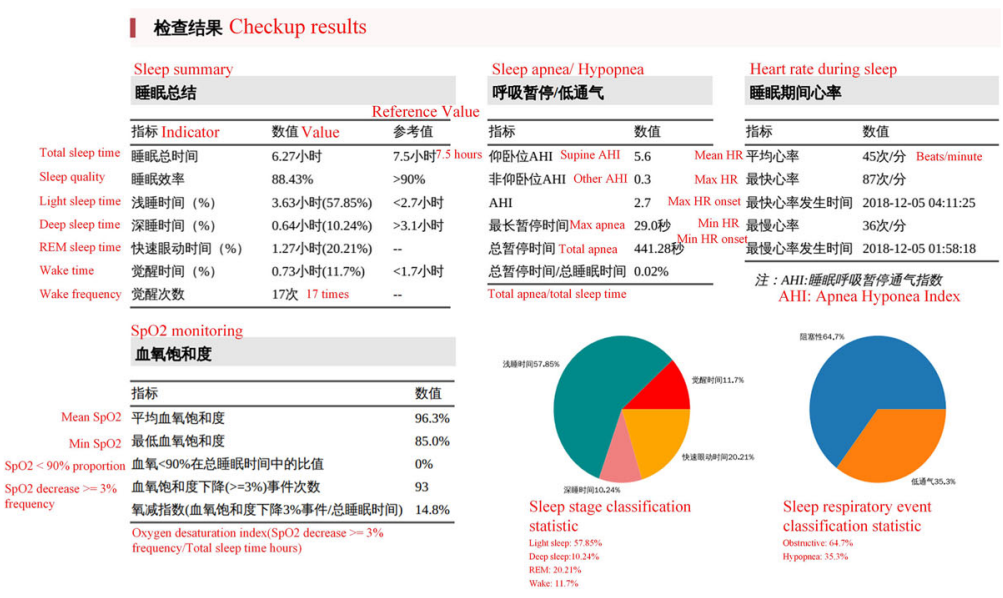

b The checkup results of the patient. AHI: Apnea Hypopnea Index

Fig. 9 A part of an example sleep quality and sleep disorder screening report. a Example patient's physiological signal trend-charts throughout the night. b The checkup results of the patient. AHI: Apnea Hypopnea Index

\section{A case study}

In order to further explain the value of the system applied in the clinic, we illustrate a case of a patient monitored by the SensEcho at the general ward of the HBO department, who was 44 years old, male, $180 \mathrm{~cm}$ height, $85 \mathrm{~kg}$ weight when admitted, and complained of chest pain for 2 years. He was diagnosed with coronary heart disease, myocardial infarction, hyperlipemia and hypertension risk level 3 . Figure 10 shows a section of the HR, RR and triaxial acceleration signals for his first night of admission, when the patient experienced a deterioration of his condition. Doctors gave first aid when the system generated alarms at about 9:15 pm. Meanwhile, we found that from 9:15 pm to 9:30 pm, the patient got two episodes of atrial fibrillation. The first one was $10 \mathrm{~min}$ ahead of the coming severe atrial fibrillation. A high frequency of premature beats occurred before 9:00 pm also. From the triaxial acceleration signals, we can find that the patient was relatively quiet before the atrial fibrillation occurred and he changed his position before the first atrial fibrillation ended. The second episode of atrial fibrillation was deadly and needed intervention immediately. Fortunately, he survived. It is believed that in this case the detailed information of disease deterioration may be missed in the absence of monitoring by the system.

\section{Discussion}

In this paper, we present a wireless physiological signal monitoring system, which is intended to provide higher dimensional information in order to realize patient health state monitoring and clinical decision support. Potential users include doctors, nurses and patients. For doctors, they receive various daily reports which contain physiological signal monitoring results, preliminary analysis results and AI-based decision support suggestions. For nurses, the system can decrease their workload. Some of the clinical practices are expected to be replaced by the system as well. For patients, the system can provide real-time multi-dimensional physiological signal monitoring, leading to better care and early warning of clinical deterioration. The system has been deployed in realistic clinical practice. Our key contributions are summarized as follows.

- SensEcho is a medical-grade wireless monitoring system, which provides comprehensive information of patients for clinicians based on signal processing technology and machine learning algorithms.

- We have deployed this system in a clinical environment and constantly update it according to the clinical feedback. The system is one of the earliest attempts to implement the physiological signal monitoring system in the clinic in China.

- The system is well integrated with the existing HIS and we provide a practical way to combine the EHRs and physiological signals.

- We explored a set of time series physiological signal acquisition, storage, and analysis processes, facilitating the follow-up research studies.

Currently, many studies on monitoring systems focus on the early warning of the risk of disease deterioration and have shown that the wearable devices have better performance than EWS, which is a commonly used clinical score $[4,6,37]$. We believe that wearable devices have the potential to provide 

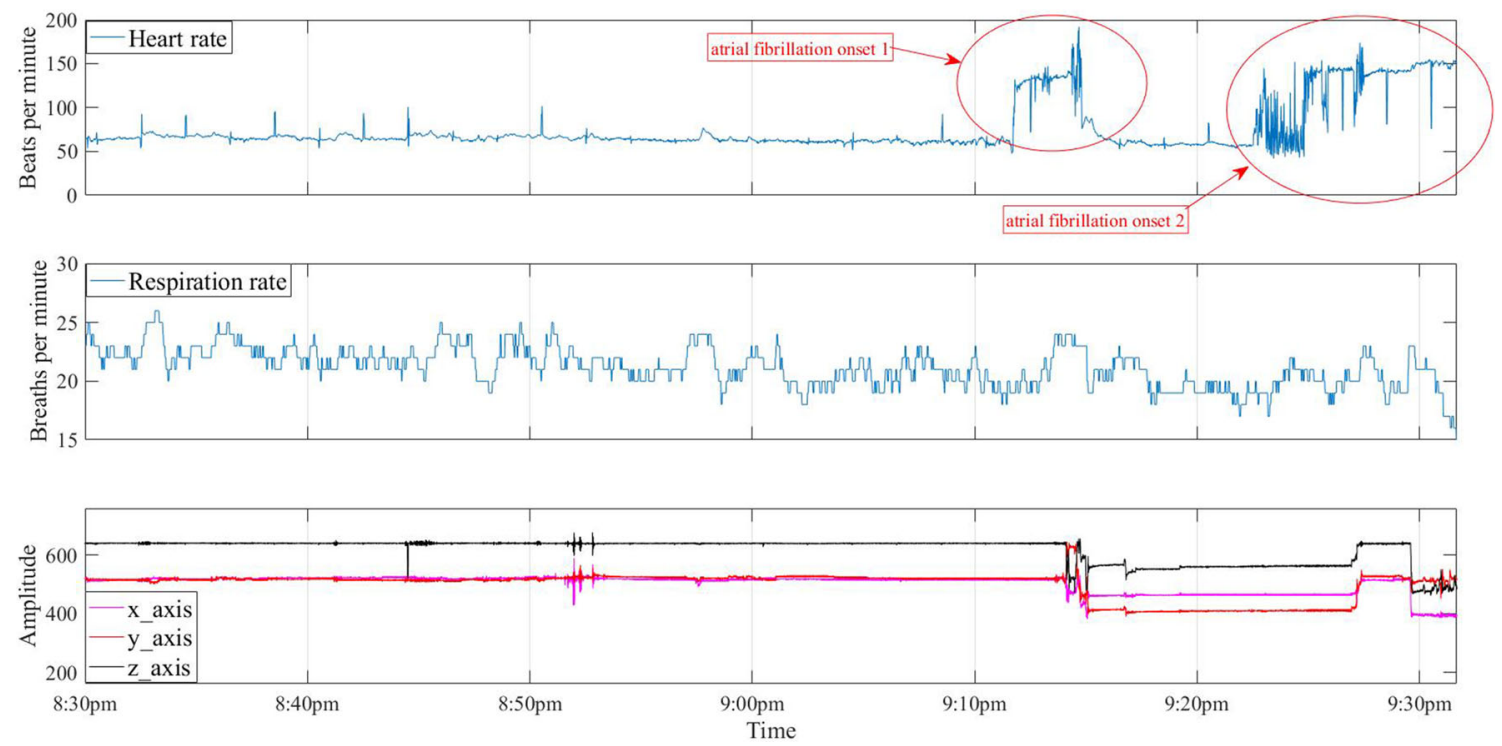

Fig. 10 Vital signals monitored by SensEcho of a patient with atrial fibrillation in HBO department

more valuable information as a clinical aid based on the analysis of physiological signals. There is a wealth of information in the reports we provided, which can help clinicians make better evaluation of patients or quantitatively evaluate the therapeutic effect. Moreover, an important prerequisite for good system operation is the guarantee of good data quality. We designed a set of schemes to ensure data quality and model performance, including training for nurses before implementation of the system, data cleaning standard operating procedure and sufficient validation of the models. To achieve a better capability and usability of the system, we need to develop and explore effective methods to reduce the impact of outliers, improve the measurement accuracy and accumulate more data to improve the model performance.

Despite the application of this system in the HBO and the Respiratory Medicine Department and its featured applications according to the clinical demands, it is believed that there is still a long way to go before the system can be used widely clinically. Furthermore, there are still some limitations to our study. One major limitation is the unfinished validation of several applications in the system. The validation study calls for the cooperation between clinical experts and engineers, which is a kind of cross-disciplinary cooperation that is often difficult but important because we need to match the engineering results with clinical experience. Another limitation of the study is the inadequate use of EHR information, although we tried the combination of EHRs and continuous physiological signals, only a fraction of EHR information has been included, and a great deal of valuable information remains to be integrated.

The future work of our study includes the further validation of the system as well as keeping going on the data mining work of the EHRs and time-series physiological signals. In addition, to our knowledge, there are three extra meaningful and interesting research focuses summarized as follows.

The first research focus is, at the time of writing, the whole world is suffering from the Corona Virus Disease (COVID19), which has a much stronger infection capacity than the flu. Patients infected with the COVID-19 are more likely to develop symptoms such as fever and respiratory distress, with their SpO2 decline severely. Clinicians are at high risk of infection when having direct contact with patients although they wear protective gear. Experience gained from the pandemic shows that little exposure to the virus can effectively reduce the chance of infection. Clinicians especially nurses, need to measure vital signs of patients several times daily, and this procedure is expected to be replaced by the wireless monitoring system. For patients, the system can monitor multidimensional physiological signals that can be used to predict and indicate the deterioration of the new coronavirus pneumonia. In conclusion, we will expand the functions and applications of the system to be more suitable for infectious disease hospitals.

The second research focus is that there are several physiological signal databases for specific use worldwide. However, we have not heard of any comprehensive database that combines the EHR information and physiological signals measured from wearable devices. Up to February 2020, we have already collected more than 1000 cases of data from the realistic clinical environment. We will clean the data and construct a comprehensive database, which will include the physiological signals, information in HIS, manually record tables from the doctors and nurses. It is time-consuming because manually recorded information is always subjective and prone to biases and errors. Data cleaning is a necessary step, and we believe that it is 
worthwhile and this database will play an important role in follow-up research.

The third research focus is that as individualized and accurate medicine is an important development of modern medicine, personalized information in physiological signals needs to be mined deeply. Continuous monitoring of physiological signals is a new type of 'Big Data' for individuals as well, reflecting the health states at different stages. The variation trend between physiological signals of one patient in different periods should be further studied to develop an index that quantitatively evaluates the health level of the patient, which can be used as a solution to individualized and accurate medicine.

Author's contributions Project design and implementation were conceived by Z.Z., D.C. and P.L. The hardware and programming were performed by M.H. Manuscript and experiment design were performed by H.X., Z.Y., W.C., X.L. and Z.W. Clinical guidance was provided by W.Y., Y.L., Y.S. and M.Y.

Funding This work was supported by NSF of China (61471398), Beijing Municipal Science and Technology (Z181100001918023), Special Grant for Healthcare (16BJZ23), CERNET Innovation Project (NGII20160701), and Big Data Research \& Development Project of Chinese PLA General Hospital (2018MBD-09), National Key Research and Development Project (2017YFC0114001), Army Logistics Study Program (ALB18R004).

\section{Compliance with ethical standards}

Conflict of interest The authors declare that they have no conflict of interest.

Ethics approval This study was approved by the ethics committee of Chinese PLA General Hospital (No. S2018-095-01).

\section{References}

1. Cardona-Morrell M, Prgomet M, Turner RM, Nicholson M, Hillman K (2016) Effectiveness of continuous or intermittent vital signs monitoring in preventing adverse events on general wards: a systematic review and meta-analysis. International Journal of Clinical Practice 70 (10):806-824

2. Watkins T, Whisman L, Booker P (2015) Nursing assessment of continuous vital sign surveillance to improve patient safety on the medical/surgical unit. Journal of Clinical Nursing 25 (1):278

3. Schein RM, Hazday N, Pena M, Ruben BH, Sprung CL (1990) Clinical antecedents to in-hospital cardiopulmonary arrest. Chest 98 (6):1388-1392. doi:https://doi.org/10.1378/chest.98.6.1388

4. Breteler MJM, KleinJan EJ, Dohmen DAJ, Leenen LPH, van Hillegersberg R, Ruurda JP, van Loon K, Blokhuis TJ, Kalkman CJ (2020) Vital Signs Monitoring with Wearable Sensors in Highrisk Surgical Patients: A Clinical Validation Study. Anesthesiology 132 (3):424-439. doi:https://doi.org/10.1097/ALN. 0000000000003029

5. Evans D, Hodgkinson B, Berry J (2001) Vital signs in hospital patients: a systematic review. 38 (6):643-650

6. Weenk M, Koeneman M, van de Belt TH, Engelen L, van Goor H, Bredie SJH (2019) Wireless and continuous monitoring of vital signs in patients at the general ward. Resuscitation 136:47-53. doi:https://doi.org/10.1016/j.resuscitation.2019.01.017

7. Baig MM, GholamHosseini H, Moqeem AA, Mirza F, Linden M (2017) A Systematic Review of Wearable Patient Monitoring Systems - Current Challenges and Opportunities for Clinical Adoption. J Med Syst 41 (7):115. doi:https://doi.org/10.1007/ s10916-017-0760-1

8. Chung M, Fortunato G, Radacsi N (2019) Wearable flexible sweat sensors for healthcare monitoring: a review. J R Soc Interface 16 (159):20190217. doi:https://doi.org/10.1098/rsif.2019.0217

9. Dinh-Le C, Chuang R, Chokshi S, Mann D (2019) Wearable Health Technology and Electronic Health Record Integration: Scoping Review and Future Directions. JMIR Mhealth Uhealth 7 (9): e12861. doi:https://doi.org/10.2196/12861

10. Heikenfeld J, Jajack A, Rogers J, Gutruf P, Tian L, Pan T, Li R, Khine M, Kim J, Wang J, Kim J (2018) Wearable sensors: modalities, challenges, and prospects. Lab Chip 18 (2):217-248. doi: https://doi.org/10.1039/c7lc00914c

11. Guillodo E, Lemey C, Simonnet M, Walter M, Baca-Garcia E, Masetti V, Moga S, Larsen M, Network H, Ropars J, Berrouiguet S (2020) Clinical Applications of Mobile Health Wearable-Based Sleep Monitoring: Systematic Review. JMIR Mhealth Uhealth 8 (4):e10733. doi:https://doi.org/10.2196/10733

12. Ramkumar PN, Haeberle HS, Ramanathan D, Cantrell WA, Navarro SM, Mont MA, Bloomfield M, Patterson BM (2019) Remote Patient Monitoring Using Mobile Health for Total Knee Arthroplasty: Validation of a Wearable and Machine LearningBased Surveillance Platform. J Arthroplasty 34 (10):2253-2259. doi:https://doi.org/10.1016/j.arth.2019.05.021

13. Duran-Vega LA, Santana-Mancilla PC, Buenrostro-Mariscal R, Contreras-Castillo J, Anido-Rifon LE, Garcia-Ruiz MA, Montesinos-Lopez OA, Estrada-Gonzalez F (2019) An IoT System for Remote Health Monitoring in Elderly Adults Through a Wearable Device and Mobile Application. Geriatrics (Basel) 4 (2). doi:https://doi.org/10.3390/geriatrics4020034

14. Bohm B, Karwiese SD, Bohm H, Oberhoffer R (2019) Effects of Mobile Health Including Wearable Activity Trackers to Increase Physical Activity Outcomes Among Healthy Children and Adolescents: Systematic Review. JMIR Mhealth Uhealth 7 (4): e8298. doi:https://doi.org/10.2196/mhealth.8298

15. Walch O, Huang Y, Forger D, Goldstein C (2019) Sleep stage prediction with raw acceleration and photoplethysmography heart rate data derived from a consumer wearable device. Sleep 42 (12). doi:https://doi.org/10.1093/sleep/zsz180

16. Monje MHG, Foffani G, Obeso J, Sanchez-Ferro A (2019) New Sensor and Wearable Technologies to Aid in the Diagnosis and Treatment Monitoring of Parkinson's Disease. Annu Rev Biomed Eng 21:111-143. doi:https://doi.org/10.1146/annurev-bioeng062117-121036

17. Sathyanarayana A, Joty S, Fernandez-Luque L, Ofli F, Srivastava J, Elmagarmid A, Arora T, Taheri S (2016) Sleep Quality Prediction From Wearable Data Using Deep Learning. JMIR Mhealth Uhealth 4 (4):e125. doi:https://doi.org/10.2196/mhealth.6562

18. Sazonova N, Browning R, Melanson E, Sazonov E (2014) Posture and activity recognition and energy expenditure prediction in a wearable platform. Conf Proc IEEE Eng Med Biol Soc 2014: 4163-4167. doi:https://doi.org/10.1109/EMBC.2014.6944541

19. Jessilyn, Dunn, Ryan, Runge, Michael, Snyder (2018) Wearables and the medical revolution. Personalized Medicine

20. Electrophysiology, T., F., o., t., E., S., o., C., t. (1996) Heart Rate Variability : Standards of Measurement, Physiological Interpretation, and Clinical Use. Circulation

21. Camm AJ, Malik M, Bigger JT, Breithardt G, Cerutti S, Cohen RJ, Coumel P, Fallen EL, Kennedy HL, Kleiger RE (1996) Heart rate variability: standards of measurement, physiological interpretation and clinical use. Task Force of the European Society of Cardiology 
and the North American Society of Pacing and Electrophysiology. Circulation 93 (5):1043-1065

22. Costa M, Goldberger AL, Peng CK (2007) Multiscale Entropy Analysis of Complex Physiologic Time Series. Physical Review Letters 89 (6):705 - 708

23. Shi P, Hu S, Yu H (2018) Recovery of heart rate variability after treadmill exercise analyzed by lagged Poincare plot and spectral characteristics. Med Biol Eng Comput 56 (2):221-231. doi:https:// doi.org/10.1007/s11517-017-1682-2

24. Gomes RL, Vanderlei LC, Garner DM, Santana MD, de Abreu LC, Valenti VE (2018) Poincare plot analysis of ultra-short-term heart rate variability during recovery from exercise in physically active men. J Sports Med Phys Fitness 58 (7-8):998-1005. doi:https://doi. org/10.23736/S0022-4707.17.06922-5

25. Li P, Yang Z, Yan W, Yan M, He M, Yuan Q, Lan K, Zheng J, Liu T, Cao D (2019) Mobicardio: A clinical-grade mobile health system for cardiovascular disease management. In: 2019 IEEE International Conference on Healthcare Informatics (ICHI). IEEE, pp 1-6

26. Lan K, Liu X, Xu H, Li P, Yang Z, Yuan Q, Zheng J, Yan W, Cao D, Zhang Z (2019) Poster: DeePTOP: Personalized Tachycardia Onset Prediction Using Bi-directional LSTM in Wearable Embedded Systems. In: Proceedings of the 2019 International Conference on Embedded Wireless Systems and Networks. pp 216-217

27. Sors A, Bonnet S, Mirek S, Vercueil L, Payen JF (2018) A convolutional neural network for sleep stage scoring from raw single-channel EEG. Biomedical Signal Processing \& Control 42 (APR.):107-114

28. Qiao, Li, Qichen, Chengyu, Liu, Supreeth, Shashikumar, Shamim, Nemati (2018) Deep learning in the cross-time frequency domain for sleep staging from a single-lead electrocardiogram. Physiological Measurement

29. Quan SF, Howard BV, Iber C, Kiley JP, Nieto FJ, O'Connor GT, Rapoport DM, Redline S, Robbins J, Samet JM (1997) The sleep heart health study: design, rationale, and methods. Sleep 20 (12): 1077-1085

30. Fonseca P, Long X, Radha M, Haakma R, Aarts RM, Rolink JM (2015) Sleep stage classification with ECG and respiratory effort. Physiological Measurement 36 (10):2027-2040

31. Meng X, Hong Y, Song J, Yang Y, Yang X (2013) Sleep stages classification based on heart rate variability and random forest. Biomedical Signal Processing \& Control 8 (6):624-633
32. Redmond SJ, Chazal PD, O'Brien C, Ryan S, Mcnicholas WT, Heneghan C (2007) Sleep staging using cardiorespiratory signals. Somnologie Schlafforschung Und Schlafmedizin 11 (4):245-256

33. Suzuki E, Yorifuji T, Ueshima K, Takao S, Sugiyama M, Ohta T, Ishikawa-Takata K, Doi H (2009) Sleep duration, sleep quality and cardiovascular disease mortality among the elderly: A populationbased cohort study. Preventive Medicine 49 (2-3):0-141

34. Strand LB, Tsai MK, Gunnell D, Janszky I, Wen CP, Chang SS (2016) Sleep duration, sleep quality and coronary heart disease mortality. International journal of cardiology 223:534

35. Ralevski E, Petrakis I, Altemus M (2019) Heart rate variability in alcohol use: A review. Pharmacol Biochem Behav 176:83-92. doi: https://doi.org/10.1016/j.pbb.2018.12.003

36. Dobbs WC, Fedewa MV, MacDonald HV, Holmes CJ, Cicone ZS, Plews DJ, Esco MR (2019) The Accuracy of Acquiring Heart Rate Variability from Portable Devices: A Systematic Review and MetaAnalysis. Sports Med 49 (3):417-435. doi:https://doi.org/10.1007/ s40279-019-01061-5

37. Breteler MJMM, Huizinga E, van Loon K, Leenen LPH, Dohmen DAJ, Kalkman CJ, Blokhuis TJ (2018) Reliability of wireless monitoring using a wearable patch sensor in high-risk surgical patients at a step-down unit in the Netherlands: a clinical validation study. BMJ Open 8 (2):e020162. doi:https://doi.org/10.1136/bmjopen2017-020162

38. Kroll RR, Mckenzie ED, Boyd JG, Sheth P, Howes D, Wood M, Maslove DM (2017) Use of wearable devices for post-discharge monitoring of ICU patients: a feasibility study. Journal of Intensive Care 5 (1):64

39. Smith GB, Prytherch DR, Meredith P, Schmidt PE, Featherstone PI (2013) The ability of the National Early Warning Score (NEWS) to discriminate patients at risk of early cardiac arrest, unanticipated intensive care unit admission, and death. Resuscitation 84 (4)

40. Bland JM, Altman DG (2007) Agreement Between Methods of Measurement with Multiple Observations Per Individual. Journal of Biopharmaceutical Statistics 17 (4):571-582

41. Steinberg C, Philippon F, Sanchez M, Fortier-Poisson P, O'Hara G, Molin F, Sarrazin JF, Nault I, Blier L, Roy K, Plourde B, Champagne J (2019) A Novel Wearable Device for Continuous Ambulatory ECG Recording: Proof of Concept and Assessment of Signal Quality. Biosensors (Basel) 9 (1). doi:https://doi.org/10. 3390/bios9010017

Publisher's Note Springer Nature remains neutral with regard to jurisdictional claims in published maps and institutional affiliations. 